\title{
Multifocal Ancient Thoracic Schwannomas: A Case Report and Review of Literature
}

\author{
Supanat Kongjarern ${ }^{\mathrm{a}}$, Pattraporn Tajarernmuang, ${ }^{\mathrm{a}}$ e, Yutthaphan Wannasopha ${ }^{\mathrm{b}}$, \\ Komson Wannasaic ${ }^{c}$, Somcharoen Saeteng ${ }^{\mathrm{d}}$
}

\begin{abstract}
Primary pleural schwannoma is a rare benign tumor of the thorax. Because of their gradual progression, they are generally asymptomatic. Herein, we report a 53-year-old male patient with a well-circumscribed mass located at the right lower lung field. He was totally asymptomatic. His contrast-enhanced computerized tomography scan showed a round, well-defined, inhomogeneous, mildly-enhancing lesion with a few small internal speckle calcifications arising from the pleura along the right lateral sixth intercostal space, measuring about $5.5 \times 4.2 \mathrm{~cm}$ in diameter, and another smaller lesion at the left apical hemithorax. Both masses were resected successfully under video-assisted thoracoscopic surgery. Histopathologic analysis revealed alternating areas of compact spindle cells and hypocellular areas and degenerative changes of blood vessels inside. Thus, the diagnosis of multifocal ancient thoracic schwannomas was established.
\end{abstract}

Keywords: Ancient schwannoma; Primary pleural schwannoma; Pleura tumor; Intercostal nerve tumor; Chest wall tumor

\section{Introduction}

Schwannoma is a benign, slow-growing tumor originating from the perineural cell named Schwann's cell. Schwannoma that arises from the intercostal nerve sheath in the pleural space is rare [1]. They are usually benign, asymptomatic and slow-

Manuscript submitted July 31, 2017, accepted August 15, 2017

aDivision of Pulmonary, Critical Care and Allergy, Department of Medicine, Faculty of Medicine, Chiang Mai University, Chiang Mai, Thailand

bepartment of Radiology, Faculty of Medicine, Chiang Mai University, Chiang Mai, Thailand

'Department of Pathology, Faculty of Medicine, Chiang Mai University, Chiang Mai, Thailand

dDepartment of Surgery, Faculty of Medicine, Chiang Mai University, Chiang Mai, Thailand

e Corresponding Author: Pattraporn Tajarernmuang, Division of Pulmonary, Critical Care and Allergy, Department of Medicine, Faculty of Medicine, Chiang Mai University, Thailand. Email: Pat_taj99@hotmail.com

doi: https://doi.org/10.14740/jmc2888w growing lesions. The malignant and multiple lesions are very rare. Multifocal lesions of thoracic schwannomas have never been reported. We report the case of bilateral pleural schwannomas in a middle-aged male with an additional brief review on the thoracic schwannoma.

\section{Case Report}

A 53-year-old male patient was referred to the pulmonary outpatient department of our tertiary hospital (Maharaj Nakorn Chiang Mai Hospital) for evaluation of an abnormal chest $\mathrm{X}$-ray from a routine checkup. He had been a smoker for 30 years. He was completely asymptomatic and the physical examination was unremarkable. His laboratory tests including complete blood count with platelet, renal, hepatic and coagulation profiles were normal.

The chest radiograph showed a round shape, well-circumscribed mass located at the right lower lung field (Fig. 1a). The contrast-enhanced computerized tomography scan showed a well-defined margin, inhomogeneous, minimal contrast enhancing lesion arising from the pleura along the right lateral sixth intercostal space, measuring about $5.5 \times 4.2 \mathrm{~cm}$ in diameter with a few small internal speckle calcifications and another smaller lesion at the left apical hemithorax (Fig. $1 b, c)$. No adjacent rib destruction, pleural effusion, or lymphadenopathy was observed. The microscopically pathological tissue from ultrasonography-guided transthoracic needle biopsy revealed a fibrotic nodule. As a consequence of multiple lesions and the uncertain nature on histopathology, the therapist team and the patient discussed and agreed to proceed to surgical resection.

On video-assisted thoracoscopic surgery, the first pleural mass was found at the right sixth intercostal space and the second one at the left apex. No pleural effusion was found. The masses were successfully resected and sent for histopathological analysis.

Grossly, the right pleural mass was red brown, rubbery with irregular external surface and the serial sections showed multi-lobulated red-brown cut surfaces with multiple hemorrhagic areas. The left pleural mass showed a piece of tan tissue. The histopathological examination reported benign schwannomas with degenerative changes (Fig. 2a, b). The patient did not receive further treatment and no evidence of recurrence or complication at the following 2 months after the operation 


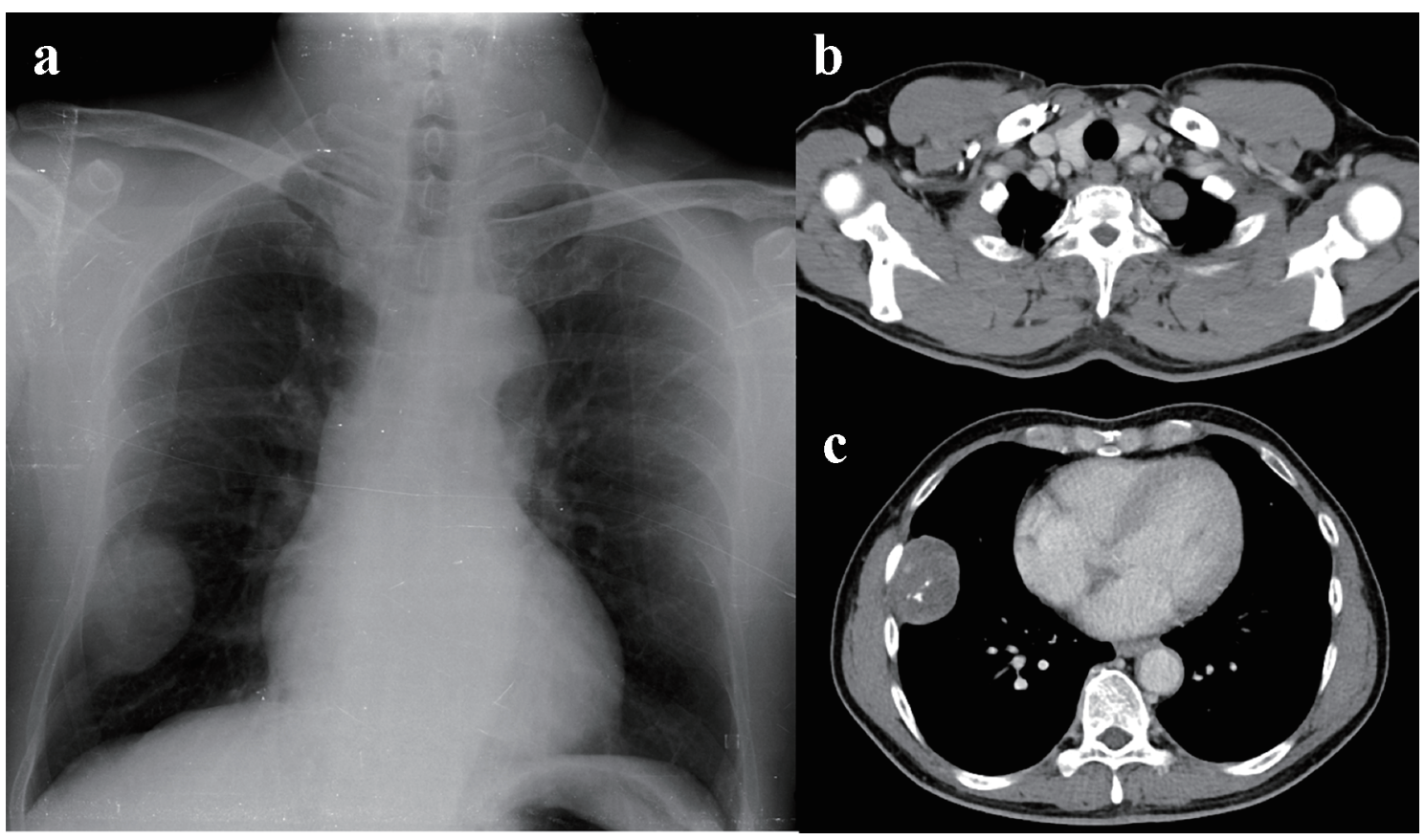

Figure 1. (a) Chest radiography showing a well-circumscribed pleural-based mass containing several small speckled calcifications along the lateral aspect of the right lower hemithorax. Also note the smaller subtle pleural-based lesion at the left apical thorax. (b, c) Axial contrast chest MDCT scan showing two slightly hypodense pleural lesions with mild enhancement at the left apical thorax (b) and right lateral lower hemithorax (c). A few small internal speckled calcifications are noted in the larger one. No adjacent rib destruction or evidence of chest wall invasion was detected.

was found.

\section{Discussion}

The primary tumor of pleura compared with metastatic tumors and malignant mesothelioma is uncommon among both children and adults. The primary pleural schwannoma, formerly called neurilemmoma, accounts for approximately $5-10 \%$ of primary chest wall tumors. They predominately appear in the para-spinal thoracic region (most common in posterior medi- astinum); however, around 5\% originate from the lateral intercostal nerves [2].

The primary pleural schwannomas are usually benign. Males and females are equally affected. Patients are mostly asymptomatic and the tumors are incidentally found on chest radiographs. Pleuritic symptoms such as pneumothorax and hemothorax that can mimic metastatic lesion of pleura rarely occur [3]. Therefore, a complete physical examination, laboratory investigation, and pathological diagnosis should be performed to discriminate benign and malignant lesions.

Generally, the chest radiograph shows a pleural based soli-
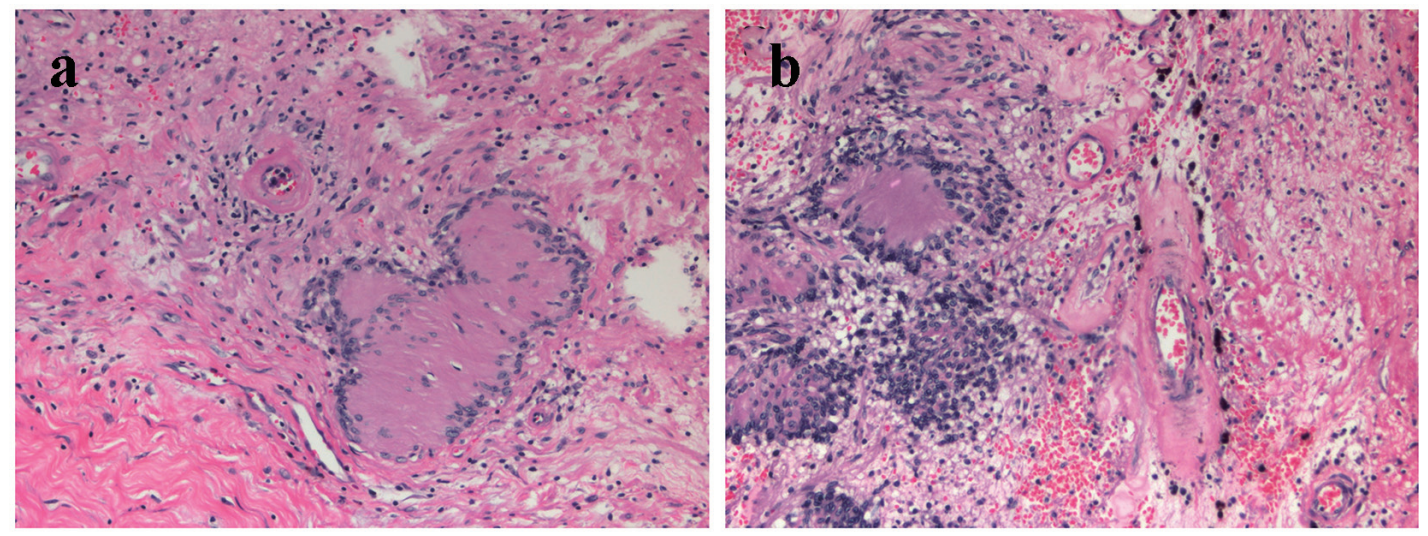

Figure 2. (a) Microscopic picture (hematoxylin and eosin staining, $\times 200$ ) showing Verocay body, formed by alternating rows of palisading nuclei and intervening nuclei-free stroma. (b) Microscopic picture (hematoxylin and eosin staining, $\times 200$ ) showing degenerative changes including hemorrhage, and hyalinization of blood vessels. 
tary mass with well-circumscribed border. Calcification, rib destruction, and pleural effusions are not common radiographic features for this disease. The computerized tomography often shows well-defined margins, iso- or slightly hypo-attenuation on plain scan and shows heterogeneous on contrast-enhanced imaging due to the distinction of cellularity, xanthomatous changes and degeneration. The signs of secondary degenerative change such as cystic formation, calcification, and rib changes are often seen in large-sized schwannomas [3].

The gross specimen of schwannoma is typically round, well encapsulated, has a firm consistency and the tan or gray color of the nerve sheath. Microscopically, schwannoma shows alternating areas of Antoni A and Antoni B. Antoni A is a highly cellular area consisting of packed long spindle cells in palisades and associated with Verocay bodies. The Antoni B pattern is fewer cells exhibiting looser stroma and myxomatous change. Additionally, degenerative changes such as cysts, hemorrhage, calcification, xanthomatous infiltration and hyalinization are the characteristics of Ancient schwannoma, a subtype of schwannoma. Immunohistochemically, the staining for S-100, a neural marker, is strongly positive. Contrastively, staining for CD-34, CD-99, bcl-2 and vimentin which are diffusely positive in the solitary fibrous tumor, should be negative [4].

Pre-operative tissue biopsy, fine-needle cytology or incisional biopsy can be used to establish the diagnosis and to determine the extent of necessary resection. The standard treatment of chest wall schwannoma is predominately surgical resection either thoracoscopically or open thoracotomy especially in the case lacking a definitive preoperative diagnosis, development of symptoms and growing tumor. Post-operative chemotherapy and radiotherapy is unnecessary. Conservative treatment with subsequent imaging can be used in high risk surgical cases, among the elderly and small-asymptomatic lesions. To the best of our knowledge, the post-operative prognosis of benign schwannoma is excellent, because the recurrence of the tumor has not been reported $[3,5]$.

\section{Funding}

None.

\section{Conflicts of Interest}

None.

\section{References}

1. Oosterwijk WM, Swierenga J. Neurogenic tumours with an intrathoracic localization. Thorax. 1968;23(4):374384.

2. McClenathan JH, Bloom RJ. Peripheral tumors of the intercostal nerves. Ann Thorac Surg. 2004;78(2):713-714.

3. Hu S, Chen Y, Wang Y, Chen KM, Song Q. Clinical and CT manifestation of pleural schwannoma. Acta Radiol. 2012;53(10):1137-1141.

4. Kale SS, Gosavi VS, Jagadale RV. Ancient chest wall schwannoma: A case report with review of literature. Med J DY Patil Univ. 2015;8:505-507.

5. Athanassiadi K, Kalavrouziotis G, Rondogianni D, Loutsidis A, Hatzimichalis A, Bellenis I. Primary chest wall tumors: early and long-term results of surgical treatment. Eur J Cardiothorac Surg. 2001;19(5):589-593. 